\title{
13. PETROLOGY OF BASALTS FROM DEEP SEA DRILLING PROJECT, LEG 38
}

\author{
W.I. Ridley, M.R. Perfit, and M-L. Adams, Lamont-Doherty Geological Observatory, \\ Columbia University, Palisades, New York
}

\section{INTRODUCTION}

We have determined the bulk composition of basalt samples drilled during Leg 38 . These data provide a basis for discussion of trace element abundances, $\mathrm{Sr}$ isotopic compositions, and mineral chemistry. Basaltic basement was collected at Sites 336, 337, 338, 342, 344, $345,348,350$. Most of the basalts examined have textures that indicate rapid cooling, evidenced by the presence of glass and skeletal-shaped olivine and plagioclase grains. Samples from Site 336 are finegrained, subophitic basalts with microphenocrysts of plagioclase and olivine. Variolitic, amygdaloidal basalt occurs at Site 337 containing sparse phenocrysts of altered olivine. Basalt at Site 338 is uniformly subophitic with occasional phenocrysts. At least two textural varieties were observed at Site 342: an upper basalt with subophitic to holocrystalline texture, and a lower basalt with a coarse diabase texture. This texture also characterizes the diabase samples examined from Site 344 . Fine-grained basalts from Site 345 are highly altered compared to fresher, aphyric samples from Sites 348 and 350 .

\section{MAJOR ELEMENT CHEMISTRY}

Major elements were determined on fused glass samples by electron microprobe. $\mathrm{Fe}_{2} \mathrm{O}_{3}$ values were computed so that $\mathrm{Fe}_{2} \mathrm{O}_{3}=0.1 \times$ total iron as $\mathrm{FeO}$. Analyses are computed on an $\mathrm{H}_{2} \mathrm{O}$-free basis. Two basalts (GAST, KNIPPA) were run as secondary standards during the analysis of the Leg 38 samples. Data are shown in Table 1.

Basalts at Site 336 are quartz tholeiites with low $\mathrm{TiO}_{2}, \mathrm{~K}_{2} \mathrm{O}$, and $\mathrm{Mg} / \mathrm{Mg}+\mathrm{Fe}\left(\mathrm{Mg}^{*}\right)$ ratios of 48 . Chemically similar basalts were analyzed from Site 337, except for slightly higher $\mathrm{Al}_{2} \mathrm{O}_{3}$ values (15\%-16\%) and higher $\mathrm{Mg}^{*}$ ratios (55). Plagioclase tholeiites occur at Site 338 with greater than $16 \% \mathrm{Al}_{2} \mathrm{O}_{3}$, low total $\mathrm{FeO}$, and high $\mathrm{Mg}^{*}$ ratios (59-63). In contrast, samples from Site 342 are more alkalic, with $3 \% \mathrm{TiO}_{2}$, somewhat higher $\mathrm{K}_{2} \mathrm{O}$, low total $\mathrm{FeO}$ and low, but variable, $\mathrm{Mg}^{*}$ ratios (45-55). Chemically similar basalt occurs at Site 343. The diabase samples at Site 344 are titania-poor olivine tholeiites, but are enriched in $\mathrm{K}_{2} \mathrm{O}$ relative to tholeiites from other sites. $\mathrm{K}_{2} \mathrm{O}$ enrichment may be due to deuteric alteration. These diabase samples also have high $\mathrm{Mg}^{*}$ ratios. One sample (344-34-2) is extremely enriched in $\mathrm{FeO}$ and $\mathrm{TiO}_{2}$, with a lesser enrichment in $\mathrm{K}_{2} \mathrm{O}$ and depletion in $\mathrm{MgO}$. We interpret this as a strongly fractionated facies of the diabase. Two samples from Site 345 are tholeiites with above average $\mathrm{MgO}$ values and high $\mathrm{Mg}^{*}$ ratios (64-70). Typical lowtitania tholeiites were analyzed from Sites 348 and 350 with $\mathrm{Mg}^{*}$ ratios between $45-51$.
The overall sample population contains both quartz tholeiites and olivine tholeiites; the latter are close to being silica oversaturated. Samples from Sites 336, 337, $338,345,350$ have bulk compositions similar to many other oceanic tholeiites, and their variations in $\mathrm{Al}_{2} \mathrm{O}_{3}$, $\mathrm{K}_{2} \mathrm{O}$, and $\mathrm{Mg}^{*}$ ratio probably reflect minor removal or addition of plagioclase and olivine together with the effects of halmyrolysis. However, those samples with high $\mathrm{Mg}^{*}$ ratios $(338,344,345)$ show no signs of excess olivine and appear to be the most primitive basalts sampled. Several samples are titania rich, but are essentially tholeiites rather than alkalic basalts. These samples also have the lowest $\mathrm{Mg}^{*}$ ratios, low $\mathrm{MgO}$, and high $\mathrm{K}_{2} \mathrm{O}$ and may represent basalt magmas that have undergone low pressure fractionation.

\section{TRACE ELEMENT CHEMISTRY}

The abundance of $\mathrm{Rb}, \mathrm{Sr}, \mathrm{Zr}$, and $\mathrm{Y}$ were determined by $\mathrm{X}$-ray fluorescence techniques and are shown in Table 2. Tholeiites from Sites 336, 337, and 338 have 1-5 ppm $\mathrm{Rb}$, which, together with low $\mathrm{K}_{2} \mathrm{O}$ contents, suggests that halmyrolysis has had little effect on basalts at these sites. $\mathrm{Y}$ contents $(22-34 \mathrm{ppm})$ and $\mathrm{Zr}$ $(50-82 \mathrm{ppm})$ are uniformly low, and together with the low $\mathrm{Sr}$ values for Site 336 and 337 basalts confirm that these samples are typical oceanic tholeiites. The $\mathrm{Sr}$ and $\mathrm{Al}_{2} \mathrm{O}_{3}$ contents of samples from Site 338 may result from minor plagioclase accumulation.

Samples from Site 342 show 2-3-fold enrichment in $\mathrm{Zr}$ and 1.5-2-fold enrichment in $\mathrm{Sr}$ over typical oceanic tholeiites, but $\mathrm{Rb}$ and $\mathrm{Y}$ are not strikingly enriched. Higher than normal $\mathrm{Zr}$ was also observed in Site 343 basalt, but $\mathrm{Rb}$ remained low. Diabases at Site 344 show marked enrichment in $\mathrm{Rb}(10-34 \mathrm{ppm})$, but this is not accompanied by enrichment in $\mathrm{Zr}$ (84-94 ppm) or $\mathrm{Y}$ (21-27 ppm), although $\mathrm{Sr}$ values are rather high (196$225 \mathrm{ppm})$.

High $\mathrm{Rb}$ and $\mathrm{Sr}$ contents also characterize Site 345 samples, but $\mathrm{Y}$ remains low $(26 \mathrm{ppm})$ and $\mathrm{Zr}$ is variable but not exceptionally high. The trace element composition of Site 348 basalts are typical of oceanic tholeiites; $\mathrm{Rb}$ is low $(1 \mathrm{ppm})$ as are $\mathrm{Sr}(88-98 \mathrm{ppm})$ and $\mathrm{Zr}(64-72$ $\mathrm{ppm})$, whereas $\mathrm{Y}$ is more varied (34-41 ppm). At Site 350 , trace element contents show no internal consistency, low $\mathrm{Rb}(4 \mathrm{ppm})$ and $\mathrm{Y}(37 \mathrm{ppm})$ are associated with high $\mathrm{Zr}(158 \mathrm{ppm})$ and $\mathrm{Sr}(250 \mathrm{ppm})$.

Generally the behavior of the lithophile (LIL) trace elements is consistent with the bulk chemistry in that samples designated as typical oceanic tholeiites show appropriate low concentrations of LIL elements. In contrast, those samples with high $\mathrm{TiO}_{2}$ contents show consistently high concentrations of $\mathrm{Zr}$ and $\mathrm{Y}$ and, in some cases, Sr. Even under the most favorable conditions of fractional crystallization, inspection of the 


\begin{tabular}{|c|c|c|c|c|c|c|c|c|c|c|c|c|c|c|c|c|c|c|c|c|}
\hline & \multicolumn{20}{|c|}{ Site (Core-Section) } \\
\hline & \multicolumn{2}{|c|}{336} & \multicolumn{3}{|c|}{337} & \multicolumn{3}{|c|}{338} & \multicolumn{3}{|c|}{342} & \multirow{2}{*}{$\begin{array}{l}343 \\
13-2\end{array}$} & \multicolumn{3}{|c|}{344} & \multicolumn{2}{|c|}{345} & \multicolumn{2}{|c|}{348} & \multirow{2}{*}{$\begin{array}{l}350 \\
16-2\end{array}$} \\
\hline & $41-1$ & $42-1$ & $13-2$ & $14-2$ & $15-2$ & $43-2$ & $43-4$ & $45-2$ & $7-2$ & $7-5$ & $8-2$ & & $34-2$ & $35-4$ & $37-2$ & $33-2$ & $35-1$ & $32-4$ & $34-2$ & \\
\hline $\mathrm{SiO}_{2}$ & 50.08 & 50.57 & 49.87 & 50.55 & 50.19 & 49.08 & 48.62 & 51.00 & 48.90 & 49.48 & 50.08 & 49.73 & 47.21 & 51.58 & 50.58 & 51.20 & 51.10 & 48.93 & 48.99 & 50.86 \\
\hline $\mathrm{TiO}_{2}$ & 1.68 & 1.62 & 1.48 & 1.36 & 1.31 & 1.38 & 1.46 & 1.52 & 2.80 & 3.18 & 3.18 & 3.05 & 3.52 & 1.46 & 1.37 & 1.83 & 2.11 & 1.60 & 1.51 & 2.67 \\
\hline $\mathrm{Al}_{2} \mathrm{O}_{3}$ & 13.95 & 14.22 & 15.94 & 15.45 & 15.11 & 16.83 & 16.69 & 16.52 & 15.42 & 15.22 & 14.81 & 14.02 & 14.10 & 15.98 & 17.46 & 17.80 & 18.38 & 15.34 & 14.87 & 14.21 \\
\hline $\mathrm{Fe}_{2} \mathrm{O}_{3}$ & 1.34 & 1.32 & 1.07 & 1.08 & 1.06 & 0.98 & 1.04 & 0.84 & 1.16 & 1.22 & 1.20 & 1.34 & 1.89 & 0.80 & 0.72 & 1.01 & 0.87 & 1.45 & 1.40 & 1.40 \\
\hline $\mathrm{FeO}$ & 10.86 & 10.69 & 8.66 & 8.77 & 8.61 & 7.93 & 8.46 & 6.87 & 9.45 & 9.94 & 9.73 & 10.83 & 15.38 & 6.51 & 5.83 & 8.17 & 7.01 & 11.75 & 11.38 & 11.41 \\
\hline $\mathrm{MnO}$ & 0.26 & 0.19 & 0.23 & 0.20 & 0.16 & 0.19 & 0.18 & 0.15 & 0.25 & 0.16 & 0.17 & 0.19 & 0.31 & 0.18 & 0.12 & 0.22 & 0.22 & 0.21 & 0.20 & 0.22 \\
\hline $\mathrm{MgO}$ & 6.40 & 6.17 & 6.61 & 6.69 & 6.70 & 7.37 & 7.57 & 7.45 & 7.24 & 5.17 & 5.11 & 5.69 & 6.65 & 7.69 & 7.00 & 9.01 & 10.24 & 6.17 & 7.53 & 5.75 \\
\hline $\mathrm{CaO}$ & 11.70 & 11.54 & 12.91 & 13.25 & 13.18 & 12.94 & 12.52 & 11.96 & 12.79 & 11.39 & 11.21 & 10.97 & 4.49 & 11.10 & 11.38 & 7.47 & 4.09 & 10.86 & 10.65 & 9.15 \\
\hline $\mathrm{Na}_{2} \mathrm{O}$ & 2.16 & 2.34 & 2.25 & 1.58 & 2.16 & 2.09 & 2.26 & 2.69 & 1.98 & 2.76 & 2.92 & 2.68 & 3.86 & 2.82 & 2.87 & 2.56 & 2.24 & 2.18 & 2.04 & 2.67 \\
\hline $\mathrm{K}_{2} \mathrm{O}$ & 0.10 & 0.09 & 0.11 & 0.10 & 0.08 & 0.13 & 0.10 & 0.10 & 0.15 & 0.31 & 0.38 & 0.23 & 0.51 & 0.47 & 0.26 & 0.73 & 0.28 & 0.11 & 0.13 & 0.22 \\
\hline $\mathrm{Fe}$ as $\mathrm{FeO}$ & 12.07 & 11.88 & 9.62 & 9.74 & 9.56 & 8.81 & 9.40 & 7.63 & 10.50 & 11.04 & 10.81 & 12.03 & 17.08 & 7.23 & 6.47 & 9.08 & 7.80 & 13.06 & 12.64 & 12.67 \\
\hline $\begin{array}{l}100 \mathrm{Mg} \\
\mathrm{Mg}+\mathrm{Fe}\end{array}$ & 48.8 & 48.3 & 55.3 & 55.2 & 55.7 & 60.1 & 59.1 & 63.7 & 55.4 & 45.7 & 45.9 & 46.0 & 41.2 & 65.6 & 66.0 & 64.1 & 70.2 & 45.9 & 51.7 & 44.9 \\
\hline \multicolumn{21}{|c|}{$\mathrm{Fe}_{2} \mathrm{O}_{3}=1 \mathrm{eO} \times 0.1$} \\
\hline $\mathrm{QZ}$ & 1.46 & 1.61 & 0.00 & 3.22 & 0.49 & 0.00 & 0.00 & 0.00 & 0.00 & 0.63 & 0.92 & 1.08 & 0.00 & 0.00 & 0.00 & 0.00 & 8.84 & 0.00 & 0.00 & 3.27 \\
\hline OR & 0.59 & 0.53 & 0.65 & 0.59 & 0.47 & 0.77 & 0.59 & 0.59 & 0.89 & 1.83 & 2.25 & 1.36 & 3.02 & 2.78 & 1.54 & 4.32 & 1.66 & 0.65 & 0.77 & 1.30 \\
\hline $\mathrm{AB}$ & 18.28 & 19.80 & 18.96 & 13.37 & 18.28 & 17.69 & 19.12 & 22.76 & 16.75 & 23.36 & 24.71 & 22.68 & 32.66 & 23.86 & 24.29 & 21.66 & 18.96 & 18.45 & 17.26 & 22.59 \\
\hline $\mathrm{AN}$ & 28.08 & 28.04 & 33.12 & 34.78 & 31.31 & 36.17 & 35.11 & 32.72 & 32.75 & 28.23 & 26.19 & 25.55 & 19.65 & 29.57 & 34.00 & 34.93 & 20.79 & 31.76 & 31.04 & 26.15 \\
\hline DI & 24.83 & 24.22 & 25.40 & 25.44 & 27.98 & 22.87 & 22.06 & 21.59 & 25.22 & 23.37 & 24.30 & 28.89 & 2.20 & 20.64 & 18.15 & 1.73 & .00 & 18.41 & 18.00 & 15.98 \\
\hline HY & 20.17 & 19.56 & 15.70 & 17.49 & 16.01 & 13.02 & 9.84 & 16.21 & 15.08 & 13.61 & 12.65 & 16.43 & 14.00 & 16.34 & 13.99 & 30.51 & 34.58 & 23.86 & 24.68 & 22.17 \\
\hline OL & 0.00 & 0.00 & 0.94 & 0.00 & 0.00 & 4.37 & 7.90 & 1.14 & 3.64 & 0.00 & 0.00 & 0.00 & 16.93 & 1.48 & 1.98 & 1.92 & .00 & 0.34 & 2.05 & 0.00 \\
\hline MT & 1.94 & 1.91 & 1.55 & 1.57 & 1.54 & 1.42 & 1.51 & 1.22 & 1.68 & 1.77 & 1.74 & 1.94 & 2.44 & 1.16 & 1.04 & 1.46 & 1.26 & 2.10 & 2.03 & 2.08 \\
\hline $\begin{array}{l}\text { IL } \\
\text { CO }\end{array}$ & 3.19 & 3.08 & 2.81 & 2.58 & 2.49 & 2.62 & 2.77 & 2.89 & 2.81 & 6.04 & 6.04 & 5.79 & 6.69 & 2.77 & 2.60 & 3.48 & $\begin{array}{l}4.01 \\
6.96\end{array}$ & 3.04 & 2.87 & 5.07 \\
\hline
\end{tabular}


TABLE 2

Trace Element Abundances in Leg 38 Basalts

\begin{tabular}{lrrrrrr}
\hline $\begin{array}{c}\text { Sample } \\
\text { (Interval in cm) }\end{array}$ & $\mathrm{Rb}$ & \multicolumn{1}{c}{$\mathrm{Sr}$} & $\mathrm{Y}$ & $\mathrm{Zr}$ & $\mathrm{Rb} / \mathrm{Sr}$ & $\mathrm{Zr} / \mathrm{Y}$ \\
\hline $336-41-1$ & 2.5 & 137 & 30 & 76 & 0.018 & 2.5 \\
$336-42-1,144-146$ & 1.0 & 144 & 35 & 82 & 0.007 & 2.3 \\
$337-13-2,140-143$ & 5 & 94 & 35 & 57 & 0.053 & 1.6 \\
$337-14-2,91-94$ & 5 & 96 & 34 & 55 & 0.053 & 1.5 \\
$337-15-2,137-140$ & 3.4 & 80 & 29 & 57 & 0.042 & 2.0 \\
$338-43-2,115-118$ & 3.3 & 167 & 23 & 62 & 0.019 & 2.7 \\
$338-43-4,54-57$ & 4.0 & 173 & 22 & 50 & 0.023 & 2.3 \\
$338-45-2,56-59$ & 1.5 & 151 & 27 & 64 & 0.010 & 2.4 \\
$342-7-2,137-140$ & 7.1 & 250 & 36 & 186 & 0.028 & 5.1 \\
$342-7-5,126-129$ & 7.8 & 241 & 42 & 180 & 0.032 & 4.3 \\
$342-8-2,65-68$ & 3.4 & 209 & 36 & 189 & 0.016 & 5.3 \\
$343-13-2,20-23$ & 5.1 & 202 & 40 & 154 & 0.025 & 3.9 \\
$344-34-2,27-30$ & 13.9 & 214 & 26 & 90 & 0.064 & 3.5 \\
$344-35-4,87-90$ & 34.1 & 196 & 22 & 84 & 0.173 & 3.8 \\
$344-37-2,135-137$ & 10.3 & 225 & 27 & 94 & 0.045 & 3.5 \\
$345-33-2,56-59$ & 15.3 & 256 & 26 & 94 & 0.059 & 3.6 \\
$345-35-1,145-148$ & 19.9 & 244 & 26 & 142 & 0.081 & 5.5 \\
$348-32-4,93-96$ & 1.6 & 98 & 41 & 72 & 0.016 & 1.8 \\
$348-34-2,107-110$ & 1.3 & 88 & 34 & 64 & 0.014 & 1.9 \\
$350-16-2,30-33$ & 3.8 & 251 & 38 & 158 & 0.015 & 4.1 \\
\hline
\end{tabular}

trace element abundances and within the constraints afforded by the major element data, it would not be possible to derive the titania-rich and LIL element-rich basalts by fractional crystallization of a typical oceanic tholeiite. Rather, it seems that these magmas may be derivatives of a compositionally distinct (and unsampled) parent magma whose mantle source was chemically different from that of typical oceanic tholeiites.

\section{MINERAL CHEMISTRY}

We have determined the range of compositions of the major mineral phases in representative samples of each basalt type from each site. Mineral determinations utilized an ARL-EMX microprobe with appropriate application of instrumental and Bence-Albee matrix corrections. Data are tabulated in Table 3 (plagioclase), Table 4 (pyroxenes), and Table 5 (iron-titanium oxides). Few samples contained fresh olivine, and no useful data could be determined for this mineral.

Generally the minerals show a limited range of compositions as a consequence of relatively rapid cooling. As might be expected, the most extensive mineral zoning was measured from Site 342 and 344 samples which are coarser grained than samples from other sites. Variations in major end-member components of pyroxenes are shown in Figure 1. The pyroxenes are dominantly calcic diopsidic augites, no low-calcium pyroxenes were detected. The zoning trends result in depletion in the wollastonite component which is not invariably associated with iron enrichment. These trends are in contrast to the iron-enrichment trends at relatively constant wollastonite content observed for many volcanic and plutonic pyroxenes. It is also noted that some of the pyroxenes fall within the two-pyroxene field boundary as defined by the trend for calcium-rich pyroxenes from the Skaergaard intrusion. In the Leg 38 samples, it appears that relatively rapid cooling initiated rapid crystal growth and consequently metastable compositional trends developed. Significant deviations from stable crystallization are also indicated by the contents of minor elements $\mathrm{Al}, \mathrm{Ti}, \mathrm{Cr}$. Rapidly cooled pyroxenes are highly aluminous and contrast

TABLE $3 \mathrm{~A}$

Plagioclase in Basalts at Site 338

\begin{tabular}{|c|c|c|c|c|c|c|c|c|c|c|c|c|c|c|c|c|}
\hline & 1 & 2 & 3 & 4 & 5 & 6 & 7 & 8 & 9 & 10 & 11 & 12 & 13 & 14 & 15 & 16 \\
\hline $\mathrm{SiO}_{2}$ & 49.46 & 49.87 & 50.88 & 47.35 & 51.32 & 51.03 & 51.45 & 52.61 & 50.89 & 51.44 & 47.42 & 47.85 & 47.36 & 47.85 & 49.44 & 48.69 \\
\hline $\mathrm{TiO}_{2}$ & 0.08 & 0.04 & 0.03 & 0.03 & 0.06 & 0.06 & 0.07 & 0.11 & 0.07 & 0.10 & 0.10 & 0.11 & 0.10 & 0.10 & 0.18 & 0.18 \\
\hline $\mathrm{Al}_{2} \mathrm{O}_{3}$ & 31.67 & 31.47 & 30.58 & 33.79 & 29.32 & 29.39 & 29.01 & 28.47 & 29.48 & 29.48 & 33.28 & 33.53 & 34.53 & 33.87 & 29.73 & 31.27 \\
\hline $\mathrm{Fe} 0$ & 0.92 & 0.79 & 0.76 & 0.57 & 1.02 & 1.31 & 1.34 & 1.14 & 0.93 & 1.08 & 0.65 & 0.56 & 0.162 & 0.59 & 1.33 & 1.36 \\
\hline $\mathrm{Mn} 0$ & 0.04 & 0.02 & 0.03 & 0.01 & 0.01 & 0.02 & 0.03 & 0.04 & 0.01 & 0.04 & 0.02 & 0.01 & 0.02 & 0.02 & 0.04 & 0.05 \\
\hline $\mathrm{MgO}$ & 0.18 & 0.21 & 0.15 & 0.17 & 0.61 & 0.60 & 0.69 & 0.66 & 0.51 & 0.55 & 0.38 & 0.35 & 0.36 & 0.34 & 0.18 & 0.13 \\
\hline $\mathrm{Ca} 0$ & 14.52 & 14.52 & 13.34 & 15.90 & 13.34 & 13.22 & 12.34 & 12.73 & 13.62 & 13.04 & 16.94 & 17.62 & 17.17 & 17.05 & 15.43 & 12.69 \\
\hline $\mathrm{Na}_{2} \mathrm{O}$ & 3.76 & 3.84 & 4.47 & 2.51 & 3.94 & 4.02 & 3.91 & 3.44 & 3.24 & 3.23 & 1.57 & 1.29 & 1.34 & 1.30 & 2.80 & 3.17 \\
\hline $\mathrm{K}_{2} \mathrm{O}$ & 0.04 & 0.04 & 0.06 & 0.02 & 0.03 & 0.06 & 0.10 & 0.06 & 0.04 & 0.10 & 0.01 & 0.02 & 0.02 & 0.01 & 0.08 & 0.31 \\
\hline Total & 100.67 & 100.80 & 100.30 & 100.35 & 99.65 & 99.72 & 98.94 & 99.26 & 98.79 & 98.85 & 100.39 & 100.92 & 101.14 & 101.13 & 99.21 & 98.08 \\
\hline $\mathrm{Si}$ & 2.256 & 2.270 & 2.320 & 2.167 & 2.352 & 2.344 & 2.371 & 2.408 & 2.348 & 2.368 & & & & & & \\
\hline $\mathrm{Ti}$ & 0.002 & 0.001 & 0.001 & 0.001 & 0.002 & 0.002 & 0.002 & 0.003 & 0.002 & 0.001 & & & & & & \\
\hline $\mathrm{Al}$ & 1.703 & 1.688 & 1.643 & 1.823 & 1.584 & 1.591 & 1.576 & 1.536 & 1.603 & 1.728 & & & & & & \\
\hline $\mathrm{Fe}$ & 0.035 & 0.030 & 0.028 & 0.021 & 0.039 & 0.043 & 0.052 & 0.003 & 0.035 & 0.022 & & & & & & \\
\hline $\mathrm{Mn}$ & 0.001 & - & 0.001 & - & - & - & 0.001 & 0.001 & - & 0.001 & & & & & & \\
\hline $\mathrm{Mg}$ & 0.012 & 0.014 & 0.009 & 0.011 & 0.041 & 0.040 & 0.047 & 0.045 & 0.035 & 0.038 & & & & & & \\
\hline $\mathrm{Ca}$ & 0.709 & 0.708 & 0.651 & 0.780 & 0.655 & 0.651 & 0.609 & 0.624 & 0.673 & 0.643 & & & & & & \\
\hline $\mathrm{Na}$ & 0.333 & 0.338 & 0.395 & 0.222 & 0.351 & 0.358 & 0.348 & 0.305 & 0.290 & 0.289 & & & & & & \\
\hline K & 0.002 & 0.002 & 0.003 & 0.001 & 0.002 & 0.003 & 0.006 & 0.003 & 0.002 & 0.006 & & & & & & \\
\hline $\mathrm{Al}+\mathrm{Si}$ & 3.959 & 3.958 & 3.963 & 3.990 & 3.936 & 3.935 & 3.947 & 3.944 & 3.951 & 4.096 & & & & & & \\
\hline An & 70 & 70 & 65 & 78 & 65 & 65 & 62 & 64 & 68 & 67 & & & & & & \\
\hline $\mathrm{Mg} / \mathrm{Fe}$ & 0.34 & 0.47 & 0.32 & 0.52 & 1.05 & 0.93 & 0.90 & 1.04 & 1.00 & 1.72 & & & & & & \\
\hline
\end{tabular}

Note: $1,2=$ microlites; $3=($ edge $), 4=($ core $)$ phenocryst $; 5-10=$ microlites; $11-14=$ weakly zoned phenocrysts; $15-16=$ microlites. 
TABLE 3B

Plagioclases in Basalts at Site $\mathbf{3 4 2}$

\begin{tabular}{|c|c|c|c|c|c|c|c|c|c|c|c|c|c|c|c|c|c|c|}
\hline & 1 & 2 & 3 & 4 & 5 & 6 & 7 & 8 & 9 & 10 & 11 & 12 & 13 & 14 & 15 & 16 & 17 & 18 \\
\hline $\mathrm{SiO}_{2}$ & 49.87 & 52.87 & 49.26 & 51.40 & 51.10 & 51.27 & 53.96 & 53.61 & 54.05 & 53.83 & 54.24 & 53.63 & $\$ 3.12$ & 50.81 & 52.41 & 21.27 & 55.52 & 51.95 \\
\hline $\mathrm{TiO}_{2}$ & 0.07 & 0.07 & 0.03 & 0.06 & 0.06 & 0.06 & 0.08 & 0.08 & 0.06 & 0.09 & 0.07 & 0.07 & 0.06 & 0.03 & 0.03 & 0.05 & 0.21 & 0.06 \\
\hline $\mathrm{Al}_{2} \mathrm{O}_{3}$ & 31.96 & 29.75 & 31.58 & 30.72 & 30.36 & 31.38 & 28.90 & 29.18 & 28.65 & 29.08 & 28.58 & 29.06 & 29.41 & 31.34 & 30.31 & 30.88 & 27.30 & 29.56 \\
\hline $\mathrm{FeO}$ & 0.71 & 0.95 & 0.67 & 0.60 & 0.67 & 0.76 & 0.85 & 1.07 & 0.72 & 0.83 & 0.87 & 0.98 & 0.80 & 0.61 & 0.59 & 0.62 & 1.65 & 0.59 \\
\hline $\mathrm{MnO}$ & - & 0.01 & 0.03 & 0.02 & 0.02 & - & 0.02 & 0.01 & 0.01 & 0.02 & 0.01 & 0.01 & 0.01 & 0.02 & 0.03 & 0.01 & - & - \\
\hline $\mathrm{MgO}$ & 0.16 & 0.10 & 0.15 & 0.17 & 0.15 & 0.12 & 0.14 & 0.11 & 0.19 & 0.19 & 0.22 & 0.08 & 0.11 & 0.18 & 0.17 & 0.13 & 0.21 & 0.18 \\
\hline $\mathrm{CaO}$ & 15.85 & 13.02 & 16.06 & 14.07 & 13.98 & 15.32 & 12.76 & 12.54 & 12.94 & 13.02 & 12.52 & 13.07 & 14.18 & 14.71 & 14.95 & 14.95 & 10.00 & 15.17 \\
\hline $\mathrm{Na}_{2} \mathrm{O}$ & 3.03 & 5.15 & 2.74 & 4.22 & 3.56 & 3.26 & 5.21 & 5,04 & 4.95 & 4.51 & 4.80 & 436 & 3.72 & 3.29 & 3.04 & 3.25 & 6.04 & 2.84 \\
\hline $\mathrm{K}_{2} \mathrm{O}$ & 0.40 & 0.75 & 0.42 & 0.42 & 0.45 & 0.43 & 0.56 & 0.56 & 0.48 & 0.44 & 0.50 & 0.65 & 0.43 & 0.36 & 0.31 & 0.32 & 0.86 & 0.31 \\
\hline Total & 102.05 & 102.67 & 100.94 & 101.70 & 100.34 & 102.60 & 102.48 & 102.20 & 102.04 & 102.01 & 101.81 & 101.91 & 101.84 & 101.35 & 101.84 & 101.48 & 101.79 & 100.66 \\
\hline Si & 2.249 & 2.364 & 2.247 & 2.317 & 1.219 & 2.294 & 2.409 & 2.399 & 2.419 & 2.406 & 2.430 & 2.410 & 2.384 & 2.295 & 2.349 & 2.312 & 2.488 & 2.376 \\
\hline $\mathrm{Ti}$ & 0.002 & 0.002 & - & 0.001 & 0.001 & 0.002 & 0.002 & 0.002 & 0.002 & 0.003 & 0.002 & 0.002 & 0.001 & 0.001 & 0.001 & 0.001 & 0.007 & 0.002 \\
\hline Al & 1.698 & 1.568 & 1.697 & 1.632 & 1.631 & 1.654 & 1.520 & 1.539 & 1.511 & 1.532 & 1.511 & 1.533 & 1.555 & 1.668 & 1.601 & 1.642 & 1.442 & 1.563 \\
\hline $\mathrm{Fe}$ & 0.026 & 0.035 & 0.025 & 0.022 & 0.025 & 0.028 & 0.031 & 0.040 & 0.027 & 0.031 & 0.032 & 0.036 & 0.030 & 0.023 & 0.022 & 0.023 & 0.062 & 0.022 \\
\hline $\mathrm{Mn}$ & - & - & 0.011 & - & - & - & - & - & - & 0.001 & - & - & - & - & 0.001 & - & - & - \\
\hline $\mathrm{Mg}$ & 0.010 & 0.007 & 0.010 & 0.011 & 0.010 & 0.008 & 0.009 & 0.007 & 0.012 & 0.013 & 0.014 & 0.005 & 0.007 & 0.012 & 0.011 & 0.009 & 0.014 & 0.012 \\
\hline $\mathrm{Ca}$ & 0.766 & 0.623 & 0.785 & 0.680 & 0.683 & 0.734 & 0.610 & 0.601 & 0.620 & 0.624 & 0.597 & 0.624 & 0.682 & 0.712 & 0.718 & 0.722 & 0.480 & 0.729 \\
\hline $\mathrm{Na}$ & 0.265 & 0.447 & 0.242 & 0.369 & 0.314 & 0.283 & 0.451 & 0.437 & 0.430 & 0.400 & 0.415 & 0.377 & 0.323 & 0.288 & 0.265 & 0.284 & 0.525 & 0.249 \\
\hline K & 0.023 & 0.043 & 0.024 & 0.024 & 0.026 & 0.024 & 0.031 & 0.032 & 0.027 & 0.025 & 0.028 & 0.037 & 0.024 & 0.020 & 0.017 & 0.018 & 0.049 & 0.017 \\
\hline $\mathrm{Al}+\mathrm{Si}_{\mathrm{i}}$ & 3.947 & 3.932 & 3.944 & 3.949 & 3.960 & 3.948 & 3.929 & 3.938 & 3.930 & 3.938 & 3.941 & 3.943 & 3.939 & 3.963 & 3.950 & 3.954 & 3.930 & 3.939 \\
\hline An & 75 & 60 & 77 & 66 & 68 & 73 & 59 & 60 & 61 & 62 & 59 & 62 & 68 & 71 & 71 & 72 & 48 & 72 \\
\hline $\mathrm{Mg} / \mathrm{Fe}_{\mathrm{e}}$ & 0.38 & 0.20 & 0.40 & 0.50 & 0.40 & 0.28 & 0.29 & 0.17 & 0.44 & 0.4 & 0.43 & 0.14 & 0.23 & 0.52 & 0.50 & 0.39 & 0.22 & 0.54 \\
\hline
\end{tabular}

Note: $1=$ (core) phenocryst; $2-6=$ zoned phenocryst; $7=($ core $)$ phenocryst; $8=($ core $)$ phenocryst; $9=($ core $)$ phenocryst; $10,11=($ core $)$ phenocryst; 12 (edge) 16 (core) $=$ zoned phenocryst; 17 (edge) 19 (core) = phenocryst; 20 (edge), 21 (core) = phenocryst; 22 (edge) -24 (core) $=$ phenocryst; $25,26=$ (core) microphenocryst: 27 (edge) 32 (core) $=$ phenocryst; 32 (core) -34 (edge) $=$ phenocryst; 35 (core), 36 (edge) $=$ phenocryst: $37,38=$ (core) phenocryst.

with the lower Al contents of more slowly cooled pyroxenes. Since the basalts show little variation in bulk $\mathrm{Al}_{2} \mathrm{O}_{3}$ content, and there is no obvious relationship between pyroxene minor element content and bulk composition, it can be concluded that the latter is not the dominant factor in determining pyroxene compositions.

A brief survey was made of plagioclase compositions in selected basalts. Results are shown in Table 3 . Plagioclase phenocrysts in Site 338 basalts have calcic

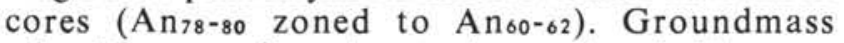
microlites range from $A n_{70}$ to $A_{n_{64}}$ and probably range down to at least $A_{60}$. Variations in phenocryst composition in Site 342 basalts suggests a complex history of plagioclase crystallization. There are two compositional groups of phenocrysts, one with $\mathrm{An}_{70}$ core composition and a second with An 60 core composition. Zoning is quite pronounced with rims of $\mathrm{An}_{44}$ composition. At Site 344 , plagioclase phenocrysts have $\mathrm{An}_{77}$ composition and may be continuously zoned to $\mathrm{An}_{33}$. This represents the most extreme compositional variability in any of the basalts analyzed.

All of the plagioclases contain minor amounts of $\mathrm{Ti}$, $\mathrm{Mg}$, and $\mathrm{Fe}$. Generally there is a positive correlation between $\mathrm{An}$ content and $\mathrm{Ti}$ and $\mathrm{Mg}$ abundances. In addition, the $\mathrm{Mg} / \mathrm{Fe}$ ratio decreases with decreasing $\mathrm{An}$ content. The highest $\mathrm{Mg} / \mathrm{Fe}$ ratios are associated with quenched plagioclase in Site 338 basalts. Probably the partitioning of $\mathrm{Mg}$ and $\mathrm{Fe}$ into the plagioclase is not an equilibrium process for these plagioclases.

We have also briefly examined the Fe-Ti oxides in these basalts. Surprisingly, most contain primary ilmenite with minor or no titanomagnetite. Analysis of co-existing ilmenite and titanomagnetite could only be accomplished in the coarse diabase at Site 342 , indicating crystallization parameters of $1020^{\circ}-1040^{\circ} \mathrm{C}$ and $\mathrm{FO}_{2}=10^{-10} \cdot{ }^{5}-10^{-11} \mathrm{~atm}$.

\section{SUMMARY}

All basaltic rocks are either quartz or olivine tholeiites which are best divided on $\mathrm{Al}_{2} \mathrm{O}_{3}$ and $\mathrm{TiO}_{2}$ contents. In terms of major elements and limited trace element abundances, samples from Sites 336, 337 and 348 are abyssal tholeiites typical of basalts dredged from active mid-oceanic ridges. Samples from Sites 338, 344 , and 345 are high-alumina tholeiites, but some have high $\mathrm{Mg} / \mathrm{Fe}$ ratios that serve to distinguish them from high-alumina abyssal tholeiites. Basalts with distinctly higher $\mathrm{K}_{2} \mathrm{O}$, $\mathrm{TiO}_{2}$, and overall greater enrichment in lithophile trace elements, occur at Sites 342, 343, and possibly 350 . Diabase at Site 344 appears to contain patches of high $\mathrm{TiO}_{2} \mathrm{FeO}$ material which may represent local segregations of in situ fractionated liquid.

Phase chemical studies indicate the ubiquity of plagioclase and pyroxene, general lack of olivine, and rarity of titanomagnetite relative to ilmenite. Compositional zoning is most pronounced in plagioclase phenocrysts which may have grown largely prior to eruption. Compositional zoning is not extensive in the pyroxenes which are all diopsidic augites, and trends are indicative of rather fast, metastable crystallization. Minor element abundances in both plagioclase $(\mathrm{Fe}$, $\mathrm{Mg}, \mathrm{Ti})$ and pyroxene ( $\mathrm{Al}, \mathrm{Ti}, \mathrm{Cr}$ ) indicate an influence of bulk mineral chemistry, but also cooling rate has had a large effect. 
TABLE 3B - Continued

\begin{tabular}{|c|c|c|c|c|c|c|c|c|c|c|c|c|c|c|c|c|c|c|c|}
\hline 19 & 20 & 21 & 22 & 23 . & 24 & 25 & 26 & 27 & 28 & 29 & 30 & 31 & 32 & 33 & 34 & 35 & 36 & 37 & 38 \\
\hline 51.00 & 56.65 & 53.72 & 55.74 & 53.66 & 53.14 & 54.94 & 55.56 & 54.50 & 51.82 & 49.92 & 49.32 & 49.26 & 53.22 & 56.70 & 53.44 & 54.15 & 53.70 & 51.60 & 53.94 \\
\hline 0.03 & 0.11 & 0.09 & 0.10 & 0.08 & 0.10 & 0.10 & 0.09 & 0.10 & 0.03 & 0.02 & 0.02 & 0.03 & 0.07 & 0.12 & 0.07 & 0.07 & 0.09 & 0.09 & 0.09 \\
\hline 30.81 & 27.70 & 29.08 & 25.86 & 29.25 & 29.97 & 28.40 & 27.89 & 27.94 & 29.90 & 31.87 & 31.87 & 31.95 & 29.60 & 27.17 & 29.49 & 28.92 & 29.40 & 29.60 & 29.66 \\
\hline 0.55 & 0.90 & 0.76 & 1.33 & 0.73 & 0.71 & 1.05 & 1.32 & 1.02 & 0.69 & 0.68 & 0.54 & 0.67 & 1.06 & 1.11 & 0.98 & 0.81 & 1.19 & 0.86 & 0.90 \\
\hline - & 0.01 & - & - & 0.01 & 0.02 & 0.01 & 0.01 & - & 0.01 & - & 0.04 & - & 0.01 & - & - & - & 0.01 & - & - \\
\hline 0.18 & 0.10 & 0.21 & 0.17 & 0.15 & 0.15 & 0.04 & 0.01 & 0.03 & 0.16 & 0.09 & 0.14 & 0.13 & 0.05 & 0.12 & 0.09 & 0.16 & 0.06 & 0.07 & 0.08 \\
\hline 15.30 & 9.04 & 13.18 & 8.83 & 13.09 & 13.47 & 12.10 & 10.16 & 11.23 & 13.59 & 15.60 & 15.90 & 15.70 & 12.94 & 10.13 & 12.63 & 12.67 & 11.91 & 13.11 & 12.11 \\
\hline 2.75 & 6.02 & 3.96 & 5.99 & 3.87 & 3.64 & 4.34 & 5.11 & 4.79 & 3.68 & 2.34 & 2.29 & 2.16 & 3.96 & 5.39 & 4.32 & 4.17 & 4.49 & 3.90 & 4.62 \\
\hline 0.32 & 0.48 & 0.31 & 0.58 & 0.30 & 0.28 & 0.57 & 0.76 & 0.48 & 0.24 & 0.21 & 0.15 & 0.16 & 0.28 & 0.49 & 0.27 & 0.22 & 0.34 & 0.21 & 0.19 \\
\hline 100.94 & 101.01 & 101.31 & 98.6 & 101.14 & 101.48 & 101.55 & 100.91 & 100.09 & 100.12 & 100.73 & 100.27 & 100.06 & 101.19 & 101.23 & 101.29 & 101.17 & 101.19 & 99.44 & 101.59 \\
\hline 2.311 & 2.529 & 2.412 & 2.553 & 2.411 & 2.382 & 2.459 & 2.496 & 2.470 & 2.359 & 2.268 & 2.253 & 2.253 & 2.395 & 2.531 & 2.401 & 2.401 & 2.430 & 2.414 & 2.411 \\
\hline 0.001 & 0.003 & 0.003 & 0.003 & 0.003 & 0.003 & 0.003 & 0.003 & 0.003 & 0.002 & - & - & 0.001 & 0.002 & 0.004 & 0.002 & 0.002 & 0.003 & 0.003 & 0.003 \\
\hline 1.645 & 1.457 & 1.539 & 1.396 & 1.549 & 1.584 & 1.498 & 1.477 & 1.493 & 1.604 & 1.706 & 1.716 & 1.722 & 1.570 & 1430 & 1.562 & 1.529 & 1.557 & 1.599 & 1.563 \\
\hline 0.021 & 0.033 & 0.028 & 0.051 & 0.027 & 0.026 & 0.039 & 0.049 & 0.038 & 0.26 & 0.025 & 0.020 & 0.025 & 0.039 & 0.041 & 0.036 & 0.030 & 0.045 & 0.033 & 0.034 \\
\hline - & - & - & - & - & - & - & - & - & - & & 0.001 & - & - & $=$ & - & - & - & - & - \\
\hline 0.012 & 0.006 & 0.014 & 0.011 & 0.010 & 0.010 & 0.002 & 0.001 & 0.002 & 0.011 & 0.006 & 0.010 & 0.009 & 0.003 & 0.008 & 0.006 & 0.011 & 0.004 & 0.001 & 0.005 \\
\hline 0.742 & 0.432 & 0.634 & 0.433 & 0.630 & 0.647 & 0.580 & 0.489 & 0.545 & 0.663 & 0.759 & 0.778 & 0.769 & 0.623 & 0.485 & 0.608 & 0.609 & 0.573 & 0.644 & 0.580 \\
\hline 0.242 & 0.521 & 0.345 & 0.532 & 0.337 & 0.317 & 0.376 & 0.445 & 0.421 & 0.325 & 0.206 & 0.203 & 0.191 & 0.345 & 0.467 & 0.376 & 0.363 & 0.391 & 0.347 & 0.401 \\
\hline 0.018 & 0.027 & 0.018 & 0.018 & 0.017 & 0.016 & 0.032 & 0.043 & 0.028 & 0.014 & 0.012 & 0.009 & 0.009 & 0.016 & 0.028 & 0.015 & 0.012 & 0.019 & 0.012 & 0.010 \\
\hline 3.956 & 3.986 & 3.951 & 4.092 & 3.960 & 3.966 & 3.957 & 3.973 & 3.963 & 3.963 & 3.974 & 3.969 & 3.975 & 3.965 & 3.961 & 3.963 & 3.959 & 3.971 & 3.964 & 3.974 \\
\hline 74 & 45 & 64 & 44 & 64 & 66 & 60 & S1 & S5 & 66 & 76 & 78 & 77 & 62 & 50 & 61 & 61 & 59 & 64 & 58 \\
\hline 0.57 & 0.18 & 0.50 & 0.21 & 0.37 & 0.38 & 0.05 & 0.02 & 0.05 & 0.42 & 0.24 & 0.50 & 0.36 & 0.08 & 0.19 & 0.16 & 0.37 & 0.09 & 0.12 & 0.15 \\
\hline
\end{tabular}

TABLE 4A

Pyroxenes in Basalts at Sites 348 and 350

\begin{tabular}{|c|c|c|c|c|c|c|c|c|c|c|c|}
\hline & \multicolumn{4}{|c|}{348} & \multicolumn{7}{|c|}{350} \\
\hline & 1 & 2 & 3 & 4 & 5 & 6 & 7 & 8 & 9 & 10 & 11 \\
\hline $\mathrm{SiO}_{2}$ & 48.20 & 49.82 & 50.84 & 49.02 & 50.18 & 52.01 & 51.18 & 51.16 & 51.09 & 52.49 & 53.27 \\
\hline $\mathrm{TiO}_{2}$ & 1.05 & 1.01 & 1.31 & 1.25 & 1.50 & 0.63 & 1.27 & 1.55 & 1.20 & 0.61 & 1.46 \\
\hline $\mathrm{Al}_{2} \mathrm{O}_{3}$ & 4.34 & 3.86 & 4.01 & 4.27 & 4.85 & 2.16 & 2.30 & 4.34 & 3.25 & 2.03 & 1.99 \\
\hline $\mathrm{Cr}_{2} \mathrm{O}_{3}$ & 0.08 & 0.13 & 0.15 & 0.14 & 0.37 & 0.20 & 0.09 & 0.24 & 0.25 & 0.21 & 0.21 \\
\hline $\mathrm{FeO}$ & 12.45 & 13.34 & 12.21 & 10.92 & 8.50 & 8.74 & 8.82 & 9.42 & 8.12 & 9.22 & 8.60 \\
\hline $\mathrm{MnO}$ & 0.49 & 0.45 & 0.46 & 0.43 & 0.25 & 0.32 & 0.31 & 0.27 & 0.24 & 0.27 & 0.26 \\
\hline $\mathrm{MgO}$ & 14.87 & 15.85 & 16.44 & 14.84 & 16.01 & 15.30 & 18.29 & 15.32 & 14.99 & 14.91 & 16.41 \\
\hline $\mathrm{CaO}$ & 18.00 & 16.52 & 16.08 & 20.20 & 19.12 & 19.06 & 16.30 & 17.86 & 19.70 & 20.61 & 18.84 \\
\hline $\mathrm{Na}_{2} \mathrm{O}$ & 0.16 & 0.24 & 0.22 & 0.27 & 0.28 & 0.17 & 0.20 & 0.30 & 0.24 & 0.17 & 0.28 \\
\hline Total & 99.64 & 101.22 & 101.72 & 101.34 & 101.12 & 98.59 & 98.76 & 100.46 & 99.08 & 100.52 & 101.32 \\
\hline $\mathrm{Si}$ & 1.827 & 1.867 & 1.865 & 1.838 & 1.839 & 1.949 & 1.906 & 1.882 & 1.907 & 1.941 & 1.938 \\
\hline $\mathrm{Ti}$ & 0.029 & 0.028 & 0.036 & 0.034 & 0.043 & 0.017 & 0.035 & 0.043 & 0.033 & 0.017 & 0.040 \\
\hline Al & 0.194 & 0.167 & 0.173 & 0.185 & 0.209 & 0.095 & 0.101 & 0.188 & 0.143 & 0.088 & 0.085 \\
\hline $\mathrm{Cr}$ & 0.002 & 0.003 & 0.004 & 0.004 & 0.010 & 0.006 & 0.002 & 0.007 & 0.007 & 0.006 & 0.006 \\
\hline $\mathrm{Fe}$ & 0.394 & 0.410 & 0.374 & 0.335 & 0.260 & 0.274 & 0.275 & 0.289 & 0.253 & 0.285 & 0.261 \\
\hline $\mathrm{Mn}$ & 0.015 & 0.014 & 0.014 & 0.013 & 0.007 & 0.010 & 0.009 & 0.008 & 0.007 & 0.008 & 0.008 \\
\hline $\mathrm{Mg}$ & 0.840 & 0.868 & 0.899 & 0.813 & 0.874 & 0.855 & 1.015 & 0.840 & 0.834 & 0.822 & 0.890 \\
\hline $\mathrm{Ca}$ & 0.731 & 0.650 & 0.632 & 0.795 & 0.751 & 0.765 & 0.650 & 0.704 & 0.787 & 0.816 & 0.734 \\
\hline $\mathrm{Na}$ & 0.012 & 0.017 & 0.015 & 0.019 & 0.020 & 0.012 & 0.015 & 0.021 & 0.017 & 0.012 & 0.020 \\
\hline $\mathrm{Al}^{\mathrm{IV}}$ & 0.173 & 0.133 & 0.135 & 0.162 & 0.161 & 0.051 & 0.094 & 0.118 & 0.093 & 0.059 & 0.062 \\
\hline $\mathrm{Al}^{\mathrm{VI}}$ & 0.021 & 0.034 & 0.038 & 0.023 & 0.048 & 0.044 & 0.007 & 0.070 & 0.050 & 0.029 & 0.023 \\
\hline$\Sigma$ Tet & 2.000 & 2.000 & 2.000 & 2.000 & 2.000 & 2.000 & 2.000 & 2.000 & 2.000 & 2.000 & 2.000 \\
\hline$\Sigma$ Cations & 4.044 & 4.024 & 4.012 & 4.036 & 4.013 & 3.983 & 4.008 & 3.982 & 3.988 & 3.983 & 3.982 \\
\hline Wo & 37 & 34 & 33 & 41 & 40 & 40 & 33 & 48 & 42 & 42 & 39 \\
\hline En & 42 & 45 & 47 & 41 & 46 & 45 & 52 & 46 & 44 & 42 & 47 \\
\hline Fs & 21 & 21 & 20 & 18 & 14 & 15 & 15 & 16 & 14 & 16 & 14 \\
\hline Quad & 88.6 & 89.3 & 88.7 & 88.3 & 86.7 & 93.1 & 92.2 & 87.2 & 90.0 & 94.1 & 92.2 \\
\hline Others & 11.4 & 10.7 & 11.3 & 11.7 & 13.3 & 6.9 & 7.8 & 12.8 & 10.0 & 5.9 & 7.8 \\
\hline
\end{tabular}

Note: $1,2=$ Phenocrysts; $3,4=$ groundmass grains; $5-7=$ center to edge of phenocrysts; $8=$ center of phenocryst; $9-11=$ center to edge of phenocrysts. 
TABLE 4B

Pyroxenes in Basalts at Site 336 and 337

\begin{tabular}{|c|c|c|c|c|c|c|c|c|}
\hline & 1 & 2 & 3 & 4 & 5 & 6 & 7 & 8 \\
\hline $\mathrm{SiO}_{2}$ & 51.26 & 52.13 & 52.13 & 52.01 & 50.05 & 51.26 & 52.13 & 52.13 \\
\hline $\mathrm{TiO}_{2}$ & 0.52 & 0.42 & 0.47 & 0.72 & 0.84 & 0.52 & 0.42 & 0.47 \\
\hline $\mathrm{Al}_{2} \mathrm{O}_{3}$ & 3.56 & 3.92 & 2.69 & 2.20 & 4.31 & 3.56 & 3.92 & 2.69 \\
\hline $\mathrm{Cr}_{2} \mathrm{O}_{3}$ & 0.09 & 0.15 & 0.17 & 0.01 & 0.13 & 0.09 & 0.15 & 0.17 \\
\hline $\mathrm{FeO}$ & 9.14 & 5.94 & 6.16 & 5.96 & 8.32 & 9.14 & 5.96 & 6.16 \\
\hline $\mathrm{MnO}$ & 0.18 & 0.19 & 0.19 & 0.21 & 0.21 & 0.18 & 0.19 & 0.19 \\
\hline $\mathrm{MgO}$ & 17.08 & 16.71 & 16.79 & 16.78 & 16.02 & 17.08 & 16.71 & 16.79 \\
\hline $\mathrm{CaO}$ & 18.07 & 20.88 & 21.20 & 20.71 & 18.08 & 18.07 & 20.88 & 21.20 \\
\hline $\mathrm{Na}_{2} \mathrm{O}$ & 0.17 & 0.17 & 0.17 & 0.22 & 0.23 & 0.17 & 0.17 & 0.17 \\
\hline Total & 100.07 & 100.51 & 99.97 & 98.82 & 98.19 & 100.07 & 100.53 & 99.97 \\
\hline $\mathrm{Si}$ & 1.892 & 1.900 & 1.916 & 1.930 & 1.879 & 1.892 & 1.900 & 1.916 \\
\hline $\mathrm{Ti}$ & 0.014 & 0.011 & 0.013 & 0.020 & 0.023 & 0.014 & 0.011 & 0.013 \\
\hline $\mathrm{Al}$ & 0.154 & 0.168 & 0.116 & 0.096 & 0.190 & 0.154 & 0.168 & 0.116 \\
\hline $\mathrm{Cr}$ & 0.002 & 0.004 & 0.005 & 0.001 & 0.004 & 0.002 & 0.004 & 0.005 \\
\hline $\mathrm{Fe}$ & 0.282 & 0.181 & 0.189 & 0.185 & 0.261 & & & \\
\hline $\mathrm{Mn}$ & 0.005 & 0.005 & 0.005 & 0.006 & 0.006 & & & \\
\hline $\begin{array}{l}\mathrm{Mg} \\
\mathrm{Ca}\end{array}$ & $\begin{array}{l}0.940 \\
0.714\end{array}$ & 0.908 & 0.920 & 0.928 & 0.896 & & & \\
\hline $\mathrm{Na}$ & 0.012 & 0.012 & 0.012 & 0.016 & 0.017 & & & \\
\hline $\mathrm{Al}^{\mathrm{IV}}$ & 0.108 & 0.100 & 0.084 & 0.070 & 0.121 & 0.108 & 0.100 & 0.084 \\
\hline $\mathrm{Al}^{\mathrm{VI}}$ & 0.046 & 0.068 & 0.032 & 0.026 & 0.069 & 0.046 & 0.068 & 0.032 \\
\hline 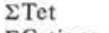 & 2.000 & 2.000 & 2.000 & 2.000 & 2.000 & 2.000 & 2.000 & 2.000 \\
\hline ¿Cations & 4.015 & 4.004 & 4.011 & 4.005 & 4.003 & & & \\
\hline Wo & 37 & 43 & 42 & 43 & 38 & & & \\
\hline En & 48 & 47 & 47 & 48 & 47 & & & \\
\hline Fs & 15 & 10 & 11 & 9 & 15 & & & \\
\hline Quad & 91.1 & 90.4 & 92.7 & 93.3 & 88.7 & & & \\
\hline Others & 8.9 & 9.6 & 7.3 & 6.7 & 11.3 & & & \\
\hline
\end{tabular}

Note: 6-8 Edge to center of phenocryst.

TABLE 4C

Pyroxenes in Basalts at Site 338

\begin{tabular}{|c|c|c|c|c|c|c|c|c|c|c|c|}
\hline & 1 & 2 & 3 & 4 & 5 & 6 & 7 & 8 & 9 & 10 & 11 \\
\hline $\mathrm{SiO}_{2}$ & 49.20 & 50.90 & 51.81 & 50.56 & 50.32 & 52.02 & 52.35 & 49.80 & 50.95 & 49.74 & 50.96 \\
\hline $\mathrm{TiO}_{2}$ & 0.54 & 0.62 & 0.69 & 0.40 & 0.69 & 0.55 & 0.56 & 0.35 & 0.40 & 0.55 & 0.53 \\
\hline $\mathrm{Al}_{2} \mathrm{O}_{3}$ & 4.93 & 4.06 & 4.01 & 3.25 & 2.99 & 2.20 & 2.33 & 5.00 & 2.57 & 3.26 & 2.99 \\
\hline $\mathrm{Cr}_{2} \mathrm{O}_{3}$ & 0.43 & 0.34 & 0.51 & 0.37 & 0.44 & 0.08 & 0.08 & 1.06 & 0.64 & 0.28 & 0.33 \\
\hline $\mathrm{FeO}$ & 6.57 & 5.79 & 5.48 & 6.80 & 5.36 & 7.92 & 9.89 & 5.98 & 5.82 & 7.08 & 7.80 \\
\hline $\mathrm{MnO}$ & 0.17 & 0.15 & 0.14 & 0.19 & 0.18 & 0.22 & 0.23 & 0.43 & 0.34 & 0.39 & 0.43 \\
\hline $\mathrm{MgO}$ & 17.32 & 17.32 & 17.93 & 19.43 & 17.73 & 17.18 & 16.58 & 16.99 & 17.71 & 17.86 & 17.57 \\
\hline $\mathrm{CaO}$ & 20.27 & 20.93 & 21.25 & 19.24 & 21.72 & 20.14 & 19.09 & 20.49 & 20.33 & 20.14 & 19.83 \\
\hline $\mathrm{Na}_{2} \mathrm{O}$ & 0.22 & 0.23 & 0.23 & 0.17 & 0.26 & 0.20 & 0.18 & 0.16 & 0.17 & 0.18 & 0.18 \\
\hline Total & 99.71 & 100.34 & 102.05 & 100.41 & 99.69 & 100.51 & 101.30 & 100.26 & 98.93 & 99.48 & 99.62 \\
\hline $\mathrm{Si}$ & 1.824 & 1.864 & 1.863 & 1.872 & 1.869 & 1.913 & 1.919 & 1.861 & 1.912 & 1.883 & 1.916 \\
\hline $\mathrm{Ti}$ & 0.015 & 0.017 & 0.018 & 0.010 & 0.018 & 0.015 & 0.015 & 0.009 & 0.011 & 0.015 & 0.015 \\
\hline Al & 0.215 & 0.175 & 0.169 & 0.137 & 0.127 & 0.095 & 0.100 & 0.220 & 0.113 & 0.145 & 0.132 \\
\hline $\mathrm{Cr}$ & 0.012 & 0.010 & 0.014 & 0.010 & 0.012 & 0.002 & 0.002 & 0.031 & 0.019 & 0.008 & 0.009 \\
\hline $\mathrm{Fe}$ & 0.203 & 0.177 & 0.165 & 0.203 & 0.162 & 0.243 & 0.303 & 0.187 & 0.182 & 0.222 & 0.214 \\
\hline Mn & 0.005 & 0.004 & 0.004 & 0.005 & 0.005 & 0.007 & 0.007 & 0.013 & 0.011 & 0.012 & 0.013 \\
\hline $\mathrm{Mg}$ & 0.956 & 0.946 & 0.961 & 1.037 & 0.954 & 0.942 & 0.906 & 0.891 & 0.935 & 0.951 & 0.928 \\
\hline $\mathrm{Ca}$ & 0.804 & 0.821 & 0.819 & 0.738 & 0.840 & 0.793 & 0.750 & 0.780 & 0.817 & 0.776 & 0.759 \\
\hline $\mathrm{Na}$ & 0.015 & 0.016 & 0.016 & 0.011 & 0.018 & 0.014 & 0.013 & 0.011 & 0.012 & 0.013 & 0.013 \\
\hline $\mathrm{Al}^{\mathrm{IV}}$ & 0.176 & 0.136 & 0.137 & 0.128 & 0.127 & 0.087 & 0.081 & 0.139 & 0.088 & 0.117 & 0.084 \\
\hline $\mathrm{Al}^{\mathrm{VI}}$ & 0.039 & 0.039 & 0.032 & 0.009 & - & 0.008 & 0.019 & 0.081 & 0.025 & 0.028 & 0.048 \\
\hline$\Sigma T e t$ & 2.000 & 2.000 & 2.000 & 2.000 & 1.996 & 2.000 & 2.000 & 2.000 & 2.000 & 2.000 & 2.000 \\
\hline ¿Cations & 4.049 & 4.030 & 4.029 & 4.025 & 4.005 & 4.024 & 4.015 & 4003 & 4.012 & 4.025 & 3.999 \\
\hline Wo & 41 & 42 & 42 & 37 & 43 & 40 & 38 & 42 & 42 & 40 & 40 \\
\hline En & 49 & 49 & 49 & 52 & 49 & 48 & 42 & 48 & 48 & 49 & 49 \\
\hline Fs & 10 & 9 & 9 & 11 & 8 & 12 & 20 & 10 & 10 & 11 & 11 \\
\hline Quad & 88.2 & 89.7 & 89.7 & 91.8 & 91.5 & 93.6 & 93.4 & 86.7 & 92.0 & 90.9 & 91.3 \\
\hline Others & 11.8 & 10.3 & 10.3 & 8.2 & 9.5 & 6.4 & 6.6 & 13.3 & 8.0 & 9.1 & 8.7 \\
\hline
\end{tabular}

Note: $1,2=$ Edge and center of phenocryst; $3=$ center of small phenocryst $; 4,5=$ edge and center of phenocryst; $6,7=$ groundmass grains; $8-11=$ phenocrysts. 
TABLE 4D

Pyroxenes in Basalts at Site 342

\begin{tabular}{|c|c|c|c|c|c|c|c|c|c|c|c|c|}
\hline & 1 & 2 & 3 & 4 & 5 & 6 & 7 & 8 & 9 & 10 & 11 & 12 \\
\hline $\mathrm{SiO}_{2}$ & 52.17 & 51.53 & 51.61 & 49.64 & 51.51 & 49.50 & 52.54 & 51.31 & 52.04 & 49.38 & 50.82 & 51.35 \\
\hline $\mathrm{TiO}_{2}$ & 0.88 & 0.88 & 1.07 & 1.13 & 0.87 & 1.07 & 0.68 & 1.06 & 0.89 & 1.09 & 1.03 & 0.88 \\
\hline $\mathrm{Al}_{2} \mathrm{O}_{3}$ & 1.89 & 2.97 & 2.55 & 3.50 & 1.63 & 1.31 & 2.11 & 2.73 & 1.82 & 2.07 & 3.39 & 2.98 \\
\hline $\mathrm{Cr}_{2} \mathrm{O}_{3}$ & 0.07 & 0.20 & 0.34 & 0.51 & 0.03 & 0.01 & 0.28 & 0.33 & 0.07 & 0.02 & 0.31 & 0.29 \\
\hline $\mathrm{FeO}$ & 8.72 & 8.55 & 9.40 & 8.91 & 10.65 & 11.65 & 9.29 & 8.22 & 11.63 & 17.73 & 8.22 & 9.42 \\
\hline $\mathrm{MnO}$ & 0.24 & 0.21 & 0.24 & 0.17 & 0.26 & 0.31 & 0.22 & 0.18 & 0.26 & 0.36 & 0.20 & 0.25 \\
\hline $\mathrm{MgO}$ & 17.19 & 16.53 & 17.33 & 17.00 & 17.14 & 16.69 & 18.20 & 16.15 & 16.33 & 12.64 & 17.57 & 17.25 \\
\hline $\mathrm{CaO}$ & 16.43 & 16.81 & 16.52 & 17.08 & 16.29 & 16.69 & 16.58 & 18.55 & 15.98 & 15.19 & 18.47 & 17.00 \\
\hline $\mathrm{Na}_{2} \mathrm{O}$ & 0.22 & 0.31 & 0.22 & 0.27 & 0.22 & 0.23 & 0.24 & 0.23 & 0.25 & 0.23 & 0.28 & 0.26 \\
\hline Total & 97.81 & 97.99 & 99.28 & 98.21 & 98.60 & 97.46 & 100.14 & 98.76 & 99.25 & 98.71 & 99.19 & 99.68 \\
\hline $\mathrm{Si}$ & 1.955 & 1.930 & 1.916 & 1.869 & 1.935 & 1.904 & 1.930 & 1.916 & 1.946 & 1.916 & 1.888 & 1.902 \\
\hline $\mathrm{Ti}$ & 0.024 & 0.024 & 0.030 & 0.032 & 0.024 & 0.031 & 0.018 & 0.029 & 0.025 & 0.032 & 0.028 & 0.024 \\
\hline $\mathrm{Al}$ & 0.083 & 0.131 & 0.000 & 0.155 & 0.072 & 0.059 & 0.091 & 0.120 & 0.080 & 0.094 & 0.148 & 0.130 \\
\hline $\mathrm{Cr}$ & 0.002 & 0.006 & 0.010 & 0.015 & 0.001 & 0.001 & 0.008 & 0.009 & 0.002 & 0.001 & 0.009 & 0.008 \\
\hline $\mathrm{Fe}$ & 0.273 & 0.267 & 0.292 & 0.280 & 0.334 & 0.374 & 0.285 & 0.256 & 0.364 & 0.575 & 0.255 & 0.291 \\
\hline Mn & 0.007 & 0.006 & 0.007 & 0.005 & 0.008 & 0.010 & 0.007 & 0.005 & 0.008 & 0.012 & 0.006 & 0.007 \\
\hline $\mathrm{Mg}$ & 0.960 & 0.922 & 0.959 & 0.954 & 0.960 & 0.957 & 0.996 & 0.899 & 0.910 & 0.731 & 0.918 & 0.953 \\
\hline $\mathrm{Ca}$ & 0.659 & 0.674 & 0.657 & 0.689 & 0.656 & 0.687 & 0.652 & 0.742 & 0.640 & 0.631 & 0.736 & 0.674 \\
\hline $\mathrm{Na}$ & 0.016 & 0.022 & 0.016 & 0.020 & 0.016 & 0.017 & 0.017 & 0.017 & 0.018 & 0.017 & 0.020 & 0.018 \\
\hline $\mathrm{Al}^{\mathrm{IV}}$ & 0.045 & 0.070 & 0.084 & 0.131 & 0.065 & 0.059 & 0.070 & 0.084 & 0.054 & 0.084 & 0.112 & 0.098 \\
\hline $\mathrm{Al}^{\mathrm{VI}}$ & 0.038 & 0.061 & 0.027 & 0.024 & 0.007 & - & 0.021 & 0.037 & 0.026 & 0.010 & 0.036 & 0.032 \\
\hline sTet & 2.000 & 2.000 & 2.000 & 2.000 & 2.000 & 1.963 & 2.000 & 2.000 & 2.000 & 2.000 & 2.000 & 2.000 \\
\hline$\Sigma$ Cations & 3.979 & 3.982 & 9.998 & 4.019 & 4.006 & 4.040 & 4.004 & 3.994 & 3.993 & 4.009 & 4.008 & 4.007 \\
\hline Wo & 35 & 36 & 34 & 36 & 34 & 34 & 34 & 39 & 33 & 32 & 38 & 35 \\
\hline En & 51 & 49 & 50 & 50 & 49 & 47 & 51 & 47 & 47 & 38 & 48 & 50 \\
\hline Fs & 14 & 15 & 16 & 14 & 17 & 19 & 15 & 14 & 20 & 30 & 14 & 15 \\
\hline Quad & 93.4 & 90.7 & 91.6 & 89.4 & 94.1 & 94.5 & 93.2 & 91.2 & 93.5 & 92.5 & 90.0 & 91.1 \\
\hline \multirow[t]{2}{*}{ Others } & 6.6 & 9.3 & 8.4 & 10.6 & 5.9 & 5.5 & 6.8 & 8.8 & 6.5 & 7.5 & 10.0 & 8.9 \\
\hline & 13 & 14 & 15 & 16 & 17 & 18 & 19 & 20 & 21 & 22 & & 23 \\
\hline $\mathrm{SiO}_{2}$ & 50.93 & 50.28 & 51.90 & 51.37 & 51.83 & 51.34 & 52.18 & 53.19 & 51.61 & 53.61 & & 52.56 \\
\hline $\mathrm{TiO}_{2}$ & 1.01 & 1.05 & 1.49 & 1.22 & 0.91 & 1.15 & 0.68 & 0.77 & 0.67 & 0.95 & & 1.02 \\
\hline $\mathrm{Al}_{2} \mathrm{O}_{3}$ & 3.16 & 3.37 & 3.14 & 2.23 & 1.90 & 2.40 & 1.87 & 2.05 & 1.99 & 3.01 & & 3.07 \\
\hline $\mathrm{Cr}_{2} \mathrm{O}_{3}$ & 0.42 & 0.28 & 0.13 & 0.09 & 0.14 & 0.22 & 0.26 & 0.26 & 0.06 & 0.33 & & 0.49 \\
\hline $\mathrm{FeO}$ & 8.30 & 7.71 & 12.73 & 11.81 & 10.59 & 8.26 & 9.04 & 9.14 & 8.70 & 8.16 & & 8.90 \\
\hline $\mathrm{MnO}$ & 0.21 & 0.20 & 0.24 & 0.27 & 0.24 & 0.23 & 0.22 & 0.19 & 0.22 & 0.18 & & 0.17 \\
\hline $\mathrm{MgO}$ & 16.50 & 16.46 & 16.14 & 15.85 & 18.62 & 14.86 & 17.33 & 17.92 & 17.59 & 16.25 & & 15.73 \\
\hline $\mathrm{CaO}$ & 19.08 & 19.95 & 16.19 & 16.66 & 16.09 & 18.99 & 17.75 & 16.95 & 17.31 & 18.98 & & 17.82 \\
\hline $\mathrm{Na}_{2} \mathrm{O}$ & 0.26 & 0.27 & 0.25 & 0.24 & 0.20 & 0.28 & 0.28 & 0.27 & 0.22 & 0.26 & & 0.26 \\
\hline Total & 99.87 & 99.57 & 102.21 & 99.74 & 100.52 & 97.73 & 99.61 & 100.74 & 98.37 & 101.13 & & 100.02 \\
\hline $\mathrm{Si}$ & 1.887 & 1.870 & 1.895 & 1.920 & 1.909 & 1.939 & 1.933 & 1.940 & 1.931 & 1.92 & & 1.933 \\
\hline $\mathrm{Ti}$ & 0.028 & 0.029 & 0.041 & 0.034 & 0.025 & 0.032 & 0.019 & 0.021 & 0.019 & 0.02 & & 0.028 \\
\hline $\mathrm{Al}$ & 0.138 & 0.148 & 0.135 & 0.098 & 0.082 & 0.106 & 0.081 & 0.088 & 0.088 & 0.12 & & 0.133 \\
\hline $\mathrm{Cr}$ & 0.012 & 0.008 & 0.003 & 0.002 & 0.004 & 0.006 & 0.007 & 0.007 & 0.001 & 0.00 & & 0.014 \\
\hline $\mathrm{Fe}$ & 0.257 & 0.240 & 0.389 & 0.369 & 0.326 & 0.261 & 0.280 & 0.279 & 0.272 & 0.24 & & 0.273 \\
\hline $\mathrm{Mn}$ & 0.006 & 0.006 & 0.007 & 0.008 & 0.007 & 0.007 & 0.007 & 0.006 & 0.007 & 0.00 & & 0.005 \\
\hline $\mathrm{Mg}$ & 0.911 & 0.913 & 0.878 & 0.883 & 1.022 & 0.837 & 0.957 & 0.974 & 0.981 & 0.88 & & 0.862 \\
\hline $\mathrm{Ca}$ & 0.757 & 0.795 & 0.633 & 0.667 & 0.635 & 0.768 & 0.704 & 0.662 & 0.694 & 0.73 & & 0.702 \\
\hline $\mathrm{Na}$ & 0.018 & 0.019 & 0.017 & 0.017 & 0.014 & 0.020 & 0.020 & 0.019 & 0.016 & 0.01 & & 0.019 \\
\hline $\mathrm{Al}^{\mathrm{IV}}$ & 0.113 & 0.130 & 0.105 & 0.080 & 0.082 & 0.061 & 0.060 & 0.069 & 0.073 & $0.06^{\circ}$ & & 0.108 \\
\hline $\mathrm{Al}^{\mathrm{VI}}$ & 0.025 & 0.018 & 0.030 & 0.018 & - & 0.045 & 0.014 & 0.028 & 0.019 & $0.05 t$ & & 0.066 \\
\hline$\Sigma$ Tet & 2.000 & 2.000 & 2.000 & 2.000 & 1.991 & 2.000 & 2.000 & 2.000 & 2.000 & 2.00 & & 2.000 \\
\hline ¿Cations & 4.014 & 4.028 & 3.998 & 3.998 & 4.024 & 4.006 & 4.008 & 3.996 & 4.009 & 3.98 & & 3.969 \\
\hline Wo & 39 & 40 & 33 & 35 & 32 & 41 & 36 & 34 & 36 & 39 & & 38 \\
\hline En & 47 & 46 & 46 & 46 & 51 & 45 & 49 & 50 & 50 & 53 & & 47 \\
\hline Fs & 14 & 14 & 21 & 19 & 17 & 14 & 15 & 16 & 14 & 18 & & 15 \\
\hline Quad & 90.5 & 90.2 & 90.3 & 92.3 & 93.7 & 90.2 & 93.5 & 93.1 & 93.6 & 90.8 & & 90.2 \\
\hline Others & 9.5 & 9.8 & 9.7 & 7.7 & 6.3 & 9.8 & 6.5 & 6.9 & 6.4 & 9.2 & & 9.8 \\
\hline
\end{tabular}

Note: $1-3=$ Edge to center, phenocryst; $4,5=$ edge to center, phenocryst; $6-8=$ edge to center, phenocryst; $9,10=$ groundmass microlites; $11,12=$ edge and center of phenocryst; $13,14=$ center and edge of phenocryst; $15=$ inclusion in plagioclase; $16-23=$ microphenocrysts. 
TABLE 4E

Pyroxenes in Basalts at Site 344

\begin{tabular}{|c|c|c|c|c|c|c|c|c|c|c|c|c|c|}
\hline & 1 & 2 & 3 & 4 & 5 & 6 & 7 & 8 & 9 & 10 & 11 & 12 & 13 \\
\hline $\mathrm{SiO}_{2}$ & 52.25 & 51.95 & 50.78 & 51.01 & 51.74 & 50.83 & 50.89 & 50.26 & 50.40 & 50.20 & 50.15 & 50.35 & 50.64 \\
\hline $\mathrm{TiO}_{2}$ & 1.11 & 1.08 & 1.11 & 1.08 & 1.08 & 0.74 & 0.62 & 0.67 & 1.32 & 0.97 & 0.91 & 1.09 & 0.99 \\
\hline $\mathrm{Al}_{2} \mathrm{O}_{3}$ & 4.00 & 4.21 & 4.90 & 4.61 & 4.04 & 2.93 & 2.85 & 2.33 & 2.90 & 2.83 & 2.01 & 1.98 & 1.91 \\
\hline $\mathrm{Cr}_{2} \mathrm{O}_{3}$ & 0.33 & 0.40 & 0.55 & 0.51 & 0.41 & 0.48 & 0.52 & 0.20 & 0.05 & 0.06 & 0.06 & 0.06 & 0.06 \\
\hline $\mathrm{FeO}$ & 4.76 & 4.94 & 5.104 & 5.54 & 5.34 & 4.44 & 4.57 & 5.35 & 9.31 & 7.99 & 13.28 & 11.73 & 12.29 \\
\hline $\mathrm{MnO}$ & 0.16 & 0.16 & 0.14 & 0.18 & 0.17 & 0.42 & 0.40 & 0.49 & 0.45 & 0.55 & 0.74 & 0.56 & 0.61 \\
\hline $\mathrm{MgO}$ & 15.95 & 16.56 & 16.28 & 15.79 & 15.96 & 17.32 & 17.18 & 17.24 & 15.66 & 16.75 & 13.48 & 14.12 & 14.11 \\
\hline $\mathrm{CaO}$ & 20.94 & 22.11 & 21.67 & 21.11 & 21.49 & 23.25 & 23.12 & 22.85 & 18.39 & 20.10 & 18.52 & 20.74 & 20.80 \\
\hline $\mathrm{Na}_{2} \mathrm{O}$ & 0.25 & 0.31 & 0.22 & 0.23 & 0.22 & 0.22 & 0.23 & 0.21 & 0.30 & 0.28 & 0.30 & 0.36 & 0.36 \\
\hline Total & 99.75 & 101.73 & 100.69 & 100.06 & 100.45 & 100.63 & 100.38 & 99.60 & 98.78 & 99.73 & 99.45 & 100.99 & 101.77 \\
\hline $\mathrm{Si}$ & 1.908 & 1.873 & 1.850 & 1.887 & 1.901 & 1.878 & 1.846 & 1.870 & 1.895 & 1.872 & 1.912 & 1.889 & 1.890 \\
\hline $\mathrm{Ti}$ & 0.030 & 0.029 & 0.030 & 0.029 & 0.029 & 0.020 & 0.016 & 0.018 & 0.037 & 0.027 & 0.026 & 0.030 & 0.027 \\
\hline $\mathrm{Al}$ & 0.172 & 0.179 & 0.210 & 0.194 & 0.171 & 0.125 & 0.122 & 0.102 & 0.128 & 0.124 & 0.090 & 0.087 & 0.084 \\
\hline $\mathrm{Cr}$ & 0.009 & 0.011 & 0.015 & 0.014 & 0.011 & 0.013 & 0.015 & 0.006 & 0.001 & 0.001 & 0.001 & 0.001 & 0.001 \\
\hline $\mathrm{Fe}$ & 0.145 & 0.149 & 0.153 & 0.165 & 0.161 & 0.134 & 0.138 & 0.166 & 0.292 & 0.249 & 0.423 & 0.368 & 0.383 \\
\hline Mn & 0.005 & 0.004 & 0.004 & 0.005 & 0.005 & 0.012 & 0.012 & 0.015 & 0.014 & 0.016 & 0.023 & 0.018 & 0.019 \\
\hline $\mathrm{Mg}$ & 0.868 & 0.889 & 0.884 & 0.842 & 0.858 & 0.935 & 0.929 & 0.956 & 0.878 & 0.931 & 0.766 & 0.790 & 0.785 \\
\hline $\mathrm{Ca}$ & 0.819 & 0.854 & 0.846 & 0.809 & 0.830 & 0.902 & 0.976 & 0.911 & 0.741 & 0.803 & 0.757 & 0.834 & 0.832 \\
\hline $\mathrm{Na}$ & 0.018 & 0.021 & 0.016 & 0.016 & 0.015 & 0.015 & 0.016 & 0.015 & 0.022 & 0.020 & 0.022 & 0.026 & 0.026 \\
\hline $\mathrm{Al}^{\mathrm{IV}}$ & 0.092 & 0.127 & 0.150 & 0.103 & 0.099 & 0.122 & 0.122 & 0.102 & 0.105 & 0.124 & 0.088 & 0.087 & 0.084 \\
\hline $\mathrm{Al}^{\mathrm{VI}}$ & 0.080 & 0.052 & 0.060 & 0.091 & 0.072 & 0.003 & - & - & 0.023 & - & 0.002 & - & - \\
\hline$\Sigma$ Tet & 2.000 & 2.000 & 2.000 & 2.000 & 2.000 & 2.000 & 1.968 & 1.972 & 2.000 & 1.996 & 2.000 & 1.976 & 1.974 \\
\hline ¿Cations & 3.974 & 4.009 & 4.008 & 3.971 & 3.981 & 4.034 & 4.070 & 4.059 & 4.008 & 4.043 & 4.020 & 4.043 & 4.047 \\
\hline Wo & 44 & 47 & 45 & 45 & 45 & 46 & 48 & 45 & 39 & 40 & 39 & 42 & 41 \\
\hline En & 47 & 48 & 47 & 46 & 46 & 47 & 45 & 47 & 46 & 47 & 39 & 40 & 39 \\
\hline Fs & 9 & 5 & 8 & 9 & 9 & 7 & 7 & 8 & 15 & 13 & 22 & 18 & 20 \\
\hline Quad & 88.6 & 88.5 & 87.2 & 87.5 & 88.8 & 91.4 & 91.8 & 92.8 & 90.4 & 91.3 & 92.3 & 92.4 & 96.7 \\
\hline Others & 11.4 & 11.5 & 12.8 & 12.5 & 11.2 & 8.6 & 8.2 & 7.2 & 9.6 & 8.7 & 7.7 & 7.6 & 3.3 \\
\hline
\end{tabular}

\begin{tabular}{|c|c|c|c|c|c|c|c|c|c|c|c|c|c|}
\hline & 14 & 15 & 16 & 17 & 18 & 19 & 20 & 21 & 22 & 23 & 24 & 25 & 26 \\
\hline $\mathrm{SiO}_{2}$ & 51.19 & 52.25 & 51.95 & 50.78 & 51.01 & 52.74 & 50.55 & 50.40 & 49.26 & 50.20 & 50.15 & 50.35 & 50.64 \\
\hline $\mathrm{TiO}_{2}$ & 0.98 & 1.11 & 1.08 & 1.11 & 1.08 & 1.08 & 1.05 & 1.32 & 1.23 & 0.97 & 0.91 & 1.09 & 0.99 \\
\hline $\mathrm{Al}_{2} \mathrm{O}_{3}$ & 1.85 & 4.00 & 4.21 & 4.90 & 4.61 & 4.04 & 2.39 & 2.90 & 3.30 & 2.83 & 2.01 & 1.98 & 1.91 \\
\hline $\mathrm{Cr}_{2} \mathrm{O}_{3}$ & 0.07 & 0.33 & 0.40 & 0.55 & 0.51 & 0.41 & 0.04 & 0.05 & 0.06 & 0.06 & 0.06 & 0.06 & 0.06 \\
\hline $\mathrm{FeO}$ & 12.89 & 4.76 & 4.95 & 5.04 & 5.54 & 5.34 & 11.62 & 9.31 & 8.02 & 7.99 & 13.28 & 11.73 & 12.29 \\
\hline $\mathrm{MnO}$ & 0.66 & 0.16 & 0.15 & 0.14 & 0.18 & 0.17 & 0.62 & 0.45 & 0.39 & 0.53 & 0.74 & 0.56 & 0.61 \\
\hline $\mathrm{MgO}$ & 13.61 & 15.96 & 16.56 & 16.28 & 15.79 & 15.96 & 13.82 & 15.66 & 16.65 & 16.75 & 13.48 & 14.12 & 14.11 \\
\hline $\mathrm{CaO}$ & 20.53 & 20.94 & 22.11 & 21.67 & 21.11 & 21.49 & 19.41 & 18.39 & 20.59 & 20.10 & 18.52 & 20.74 & 20.80 \\
\hline $\mathrm{Na}_{2} \mathrm{O}$ & 0.34 & 0.25 & 0.31 & 0.22 & 0.23 & 0.22 & 0.34 & 0.30 & 0.26 & 0.28 & 0.30 & 0.36 & 0.36 \\
\hline Total & 102.12 & 99.76 & 101.72 & 100.69 & 100.06 & 101.45 & 99.84 & 98.78 & 99.76 & 99.71 & 99.45 & 100.99 & 101.77 \\
\hline $\mathrm{Si}$ & 1.905 & 1.908 & 1.878 & 1.840 & 1.897 & 1.901 & 1.879 & 1.895 & 1.812 & 1.872 & 1.912 & 1.889 & 1.890 \\
\hline $\mathrm{Ti}$ & 0.027 & 0.030 & 0.029 & 0.030 & 0.029 & 0.029 & 0.030 & 1.037 & 0.035 & 0.027 & 0.026 & 0.030 & 0.027 \\
\hline $\mathrm{Al}$ & 0.081 & 0.172 & 0.179 & 0.210 & 0.194 & 0.171 & 0.109 & 0.128 & 0.149 & 0.124 & 0.090 & 0.087 & 0.084 \\
\hline $\mathrm{Cr}$ & 0.002 & 0.009 & 0.011 & 0.015 & 0.014 & 0.011 & 0.001 & 0.001 & 0.002 & 0.001 & 0.001 & 0.001 & 0.001 \\
\hline $\mathrm{Fe}$ & 0.401 & 0.145 & 0.149 & 0.153 & 0.165 & 0.161 & 0.376 & 0.292 & 0.257 & 0.249 & 0.423 & 0.368 & 0.383 \\
\hline Mn & 0.020 & 0.005 & 0.004 & 0.004 & 0.005 & 0.005 & 0.020 & 0.014 & 0.012 & 0.016 & 0.023 & 0.018 & 0.019 \\
\hline $\mathrm{Mg}$ & 0.755 & 0.868 & 0.889 & 0.884 & 0.842 & 0.858 & 0.797 & 0.878 & 0.951 & 0.931 & 0.766 & 0.790 & 0.785 \\
\hline $\mathrm{Ca}$ & 0.818 & 0.819 & 0.854 & 0.846 & 0.809 & 0.830 & 0.805 & 0.741 & 0.845 & 0.803 & 0.757 & 0.834 & 0.832 \\
\hline $\mathrm{Na}$ & 0.024 & 0.018 & 0.021 & 0.016 & 0.016 & 0.015 & 0.025 & 0.022 & 0.019 & 0.020 & 0.022 & 0.026 & 0.026 \\
\hline $\mathrm{Al}^{\mathrm{IV}}$ & 0.081 & 0.092 & 0.122 & 0.150 & 0.103 & 0.099 & 0.109 & 0.105 & 0.149 & 0.124 & 0.088 & 0.087 & 0.084 \\
\hline $\mathrm{Al}^{\mathrm{VI}}$ & - & 0.080 & 0.057 & 0.060 & 0.091 & 0.072 & - & 0.023 & - & - & 0.002 & - & - \\
\hline$\Sigma$ Tet & 1.986 & 2.000 & 2.000 & 2.000 & 2.000 & 2.000 & 1.988 & 2.000 & 1.961 & 1.996 & 2.000 & 1.976 & 1.974 \\
\hline$\Sigma$ Cations & 4.033 & 3.974 & 4.014 & 4.008 & 3.971 & 3.981 & 4.042 & 4.008 & 4.082 & 4.043 & 4.020 & 4.043 & 4.047 \\
\hline Wo & 41 & 45 & & 45 & 44 & 45 & 41 & 39 & 41 & 40 & 39 & 42 & 41 \\
\hline En & 38 & 47 & 47 & 47 & 46 & 46 & 40 & 46 & 46 & 47 & 39 & 39 & 39 \\
\hline Fs & 21 & 8 & 8 & 8 & 10 & 9 & 19 & 15 & 13 & 13 & 22 & 19 & 20 \\
\hline Quad & 92.7 & 88.6 & 88.5 & 87.2 & 87.5 & 88.8 & 91.4 & 90.4 & 90.4 & 91.3 & 92.3 & 92.4 & 92.7 \\
\hline Others & 7.3 & 11.4 & 11.5 & 12.8 & 12.5 & 11.2 & 8.6 & 9.6 & 9.6 & 8.7 & 7.7 & 7.6 & 7.3 \\
\hline
\end{tabular}

Note: $1-3=$ Edge-center of phenocryst; $4,5=$ center of phenocryst; $6-8=$ center-edge of phenocryst; $9,10=$ center of phenocrysts; $11-14=$ individual phenocrysts; $15-19=$ center of phenocrysts; $20-22=$ center to edge of phenocryst; $22-26=$ center to edge of phenocryst. 
TABLE 5

Spinels in Leg 38 Basalts

\begin{tabular}{|c|c|c|c|c|c|c|c|}
\hline & \multicolumn{7}{|c|}{ Site } \\
\hline & 342 & 342 & 342 & 342 & 342 & 344 & 344 \\
\hline $\mathrm{TiO}_{2}$ & 23.32 & 49.50 & 24.01 & 21.87 & 49.51 & 47.54 & 47.56 \\
\hline $\mathrm{Al}_{2} \mathrm{O}_{3}$ & 1.47 & 0.14 & 0.99 & 1.69 & 0.16 & 0.11 & 0.22 \\
\hline $\mathrm{Cr}_{2} \mathrm{O}_{3}$ & 0.06 & 0.03 & 0.08 & 0.07 & - & 0.05 & 0.07 \\
\hline $\mathrm{FeO}$ & 72.88 & 49.75 & 71.00 & 72.84 & 48.64 & 51.01 & 49.16 \\
\hline $\mathrm{MnO}$ & 0.35 & 0.36 & 0.28 & 0.47 & 0.39 & 0.46 & 0.61 \\
\hline $\mathrm{MgO}$ & 0.85 & 1.28 & 0.43 & 0.75 & 1.76 & 0.49 & 2.27 \\
\hline Total & 98.93 & 101.05 & 96.79 & 97.69 & 100.46 & & \\
\hline $\mathrm{FeO}$ & 51.69 & & 52.3 & 50.4 & & & \\
\hline $\mathrm{Fe}_{2} \mathrm{O}_{3}$ & 23.36 & & 20.8 & 24.6 & & & \\
\hline Total & 101.10 & & 98.89 & 99.85 & & & \\
\hline$\%$ Ulv. & 54.3 & & 69.7 & 63.9 & & & \\
\hline$\% \mathrm{R}_{2} \mathrm{O}_{3}$ & & 8.2 & & & 8.5 & & \\
\hline \multicolumn{8}{|c|}{ Ilmenite Basis } \\
\hline $\mathrm{FeO}$ & 38.16 & 41.83 & 38.2 & 37.5 & 40.96 & 41.04 & 38.01 \\
\hline $\mathrm{Fe}_{2} \mathrm{O}_{3}$ & 38.72 & 8.32 & 36.8 & 39.7 & 8.48 & 10.56 & 11.36 \\
\hline Total & 102.93 & 101.45 & 100.79 & 102.05 & 101.26 & 100.25 & 100.12 \\
\hline$-\log \mathrm{fO}_{2}$ & & 10.5 & & -11.0 & & & \\
\hline $\mathrm{T}^{\circ} \mathrm{C}$ & & 1040 & & 1020 & & & \\
\hline
\end{tabular}
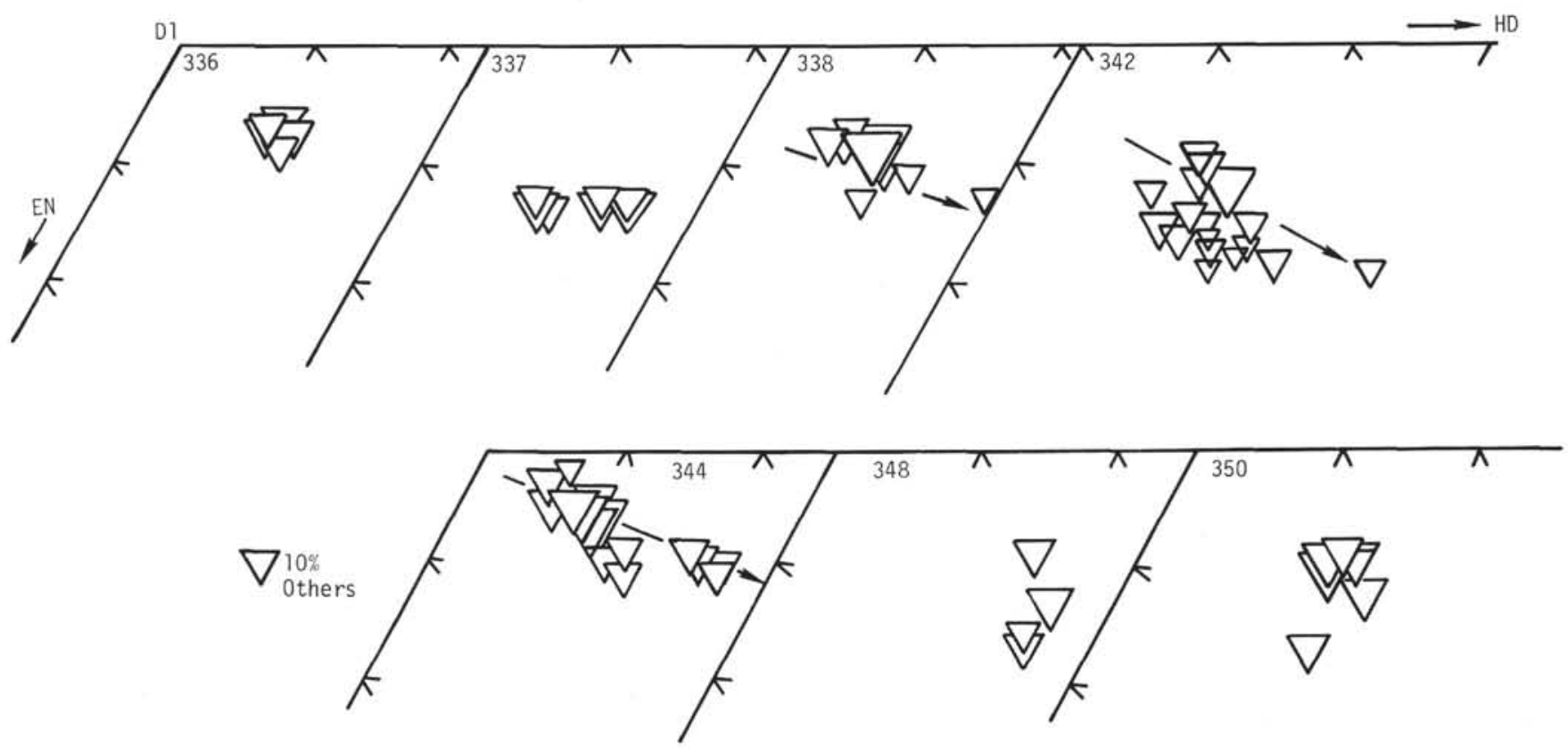

Figure 1. Pyroxene compositions in Leg 38 basalts in terms of quadrilateral components diopside (DI)-enstatite (EN)-ferrosilite (FS)-hedenbergite (HD). The size of the triangles is an indication of the amount of components other than the above present in the pyroxene. These "other" components are due to solid solutions of Al, Cr, and Ti in the pyroxene structure. 